Ciencia y Salud, Vol. III, No. 1, enero-abril, 2019 • ISSN: 2613-8816 | E-ISSN: 2613-8824

DOI: https://doi.org/10.22206/cysa.2019.v3i1.pp51-57

\title{
EVALUACIÓN DEL CONSUMO DE ANTIBIÓTICOS CON Y SIN PRESCRIPCIÓN MÉDICA EN INFANTES MENORES DE 5 AÑOS EN LA COMUNIDAD DE PAYA EN MEDIO-BANÍ, DURANTE EL PERÍODO AGOSTO - OCTUBRE 2018
}

\section{Evaluation of the consumption of antibiotics with and without a prescription in infants under 5 years of age in the community of Paya en medio, municipality of Baní, province Peravia, Dominican Republic during the period Aug-Oct 2018}

\section{Cristhian J. Almonte Quiñones*, Penélope Martínez Jiménez*, Cástulo J. Valdez Aquino*, Cristina Contreras**, Emilton López ${ }^{* *}$}

Fecha de recibido: septiembre 6, 2018 • Fecha de aprobado: noviembre 4, 2018

Cómo citar: Almonte Quiñones CJ, Martínez Jiménez P, Valdez Aquino CJ, Contreras C, López E. Evaluación del consumo de antibióticos con y sin prescripción médica en infantes menores de 5 años en la comunidad de Paya en medio-Baní, durante el período agosto-octubre 2018. cysa [Internet]. 11 abr. 2019 [citado 12 abr. 2019];1(1):51-7. Available from: https://revistas.intec. edu.do/index.php/cisa/article/view/1331

\section{Resumen}

Introducción: la resistencia antibiótica se está perfilando como una grave amenaza en todo el mundo. Cada día están propagándose a nivel mundial nuevos mecanismos de resistencia que ponen en peligro nuestra capacidad para tratar las enfermedades infecciosas.

Objetivo: evaluar el consumo de antibióticos con y sin prescripción médica de los niños menores de 5 años en la comunidad de Paya en medio-Baní, durante el periodo agosto-octubre 2018.

Material y métodos: estudio prospectivo, descriptivo, observacional, de corte transversal con una muestra de 68 niños menores de 5 años.

Resultados: se determinó que el $46 \%$ de la muestra utilizó antibióticos en los últimos 3 meses. El $68 \%$ de los infantes que consumieron antibióticos lo obtuvieron bajo prescripción médica y el $32 \%$ lo obtuvieron por venta libre. El antibiótico de mayor consumo fue la Amoxicilina. El motivo que mayor uso

\footnotetext{
*Médicos Internos. Hospital de Paya, Baní, República Dominicana. Email: cjac_24@hotmail.com;

martinezjpenelope@gmail.com;

castulojr@otlook.com

**Instituto Tecnologico de Santo Domingo, República Dominicana. Email: crismi117@hotmail.com

**Médico Pediatra. Email: emiltonlopez@hotmail.com
}

de antibiótico requirió fue relacionado a síntomas del aparato respiratorio.

Conclusiones: en un corto período de tiempo de tan solo 3 meses, menores de 5 años fueron altamente expuestos a antibióticos. Debido a que su uso puede contribuir a la resistencia bacteriana, estos resultados tienen implicaciones importantes para la educación en salud pública y para la normativa de ventas de antibióticos en la República Dominicana.

Palabras claves: Antibióticos; Prescripción médica; Pediatría; Resistencia Antibiótica; Atención primaria de salud

\section{Abstract}

Introduction: resistance is emerging as a serious threat throughout the world. Every day new mechanisms of resistance that threaten our ability to treat infectious diseases are being produced and spread worldwide.

Methods: a cross-sectional community-based survey was executed in Paya en medio-Baní of the Peravia province, a structured interview was applied to collect the data of the total population, the representatives of 67 children under 5 years, it was a retrospective taking as parameter the use of antibiotics during the last 3 months.

Results: it was determined that $46 \%$ of the sample used antibiotics in the last 3 months. $68 \%$ of the infants who consumed antibiotics obtained it under medical prescription and $32 \%$ obtained it by free sale. The most commonly used antibiotic was Amoxicillin. The reason of the 
highest use of antibiotic was related to symptoms of the respiratory system.

Conclusion: in a short period of only 3 months, children under 5 years were highly exposed to antibiotics, because their use can contribute to bacterial resistance, these results have important implications for public health education and regulations of antibiotic sales in the Dominican Republic.

Keywords: Antibiotics; prescription; pediatrics; antibiotics resistance; primary care

\section{Introducción}

La resistencia a los antibióticos se está perfilando como una grave amenaza en todo el mundo. Cada día están produciéndose y propagándose a nivel mundial nuevos mecanismos de resistencia que ponen en peligro nuestra capacidad para tratar las enfermedades infecciosas comunes. Enfermedades como la neumonía, la tuberculosis, la sepsis, la gonorrea o las enfermedades de transmisión alimentaria, son cada vez más difíciles e incluso pueden tornarse imposibles de tratar, hecho justificado por la pérdida de la eficacia de los antibióticos.

Sin antimicrobianos eficaces para la prevención y el tratamiento de infecciones, los procedimientos médicos como el trasplante de órganos, la quimioterapia contra el cáncer, el tratamiento de la diabetes o cirugías mayores (por ejemplo, cesáreas o reemplazos de cadera) se vuelven de alto riesgo. (1)

No poner este tema sobre la mesa de discusión y no tomar medidas urgentes, encamina al mundo a vivir en una era post-antibióticos en la que muchas infecciones comunes y lesiones menores volverán a ser potencialmente mortales.

Los antibióticos eliminan a los competidores sensibles a las drogas, dejando atrás bacterias resistentes para reproducirse como resultado de la selección natural. A pesar de las advertencias sobre el uso excesivo, los antibióticos se recetan en exceso en todo el mundo. (2)
Es necesario que se cambie urgentemente la forma de prescribir y utilizar los antibióticos. Aunque se desarrollen nuevos medicamentos, si no se modifican los comportamientos actuales, la resistencia a los antibióticos seguirá representando una grave amenaza. Los cambios de comportamiento también deben incluir medidas destinadas a reducir la propagación de las infecciones, a través de la vacunación, el lavado de las manos, la seguridad de las relaciones sexuales y una buena higiene alimentaria. (3)

Es necesario considerar el problema de la automedicación cuando se exploran los factores que influyen en el uso indebido de antibióticos. Bi et al. encontraron que casi el 59\% de los niños en China tenían automedicación de sus padres. (4) La automedicación es más común en los países en desarrollo debido a factores como la disponibilidad inmediata de antibióticos sin receta médica, el acceso irrestricto a antibióticos, la falta de regulación sobre medicamentos y los médicos y farmacéuticos que prescriben y dispensan antibióticos independientemente de la causa de la infección. Una encuesta por Kogan, et al., 1994 en Estados Unidos mostró que el 54\% de los niños tenían automedicación parental.

La resistencia a los antibióticos es una emergencia para la salud mundial que comprometerá gravemente el avance de la medicina moderna (5). Hace que se incrementen los costos médicos, que se prolonguen las estancias hospitalarias y que aumente la mortalidad de los pacientes.

Desafortunadamente, a pesar de que la necesidad ha crecido en los últimos años, el desarrollo de nuevos antibióticos ha disminuido, los objetivos terapéuticos moleculares más vulnerables de las bacterias han sido caracterizados y estudiados, pendiente de la identificación de nuevos objetivos terapéuticos nos encontramos en una década en la que solamente contamos con los antibióticos que tenemos disponibles. (6) 


\section{Materiales y métodos}

Esta investigación fue de carácter prospectivo, realizada durante el período agosto-septiembre 2018; descriptivo, debido a que se detallaron las características de las variables, observacional debido a que no se modificaron las mismas y de corte transversal, debido a que las variables se estudiaron en una sola ocasión y no se les dará seguimiento en el tiempo.

Los datos fueron recolectados mediante una encuesta transversal basada en la comunidad elaborada por los autores junto al coordinador, donde se utilizaron preguntas validadas de estudios previos. El cuestionario fue aplicado a la madre, padre o tutor de los nińos menores de 5 ańos de la comunidad de Paya abajo (Ver anexo \#1). En el mismo se evaluaron las siguientes variables: el consumo de antibióticos según edad, sexo, los motivos que requirieron su uso, los antibióticos más utilizados y su forma de presentación, el método de adquisición, tiempo de uso y la percepción de efectividad de estos.

Se tomó un grupo control al cual se le aplicó la encuesta y posteriormente se revisó el mismo con el objetivo de validar el entendimiento de las preguntas por parte de los entrevistados. Los investigadores visitaron los hogares y explicaron a los padres o tutores el propósito de la investigación para verificar si accedían a participar, se les preguntó el significado de la palabra "antibióticos" para confirmar su entendimiento sobre el tema, si la definición era aceptable se procedía a aplicar la encuesta.

La población de este estudio estuvo compuesta por 68 niños menores de 5 años de la comunidad de Paya en medio, Baní, siendo el universo todos los habitantes de Paya en medio. La muestra estuvo compuesta por la totalidad de la población.

Como criterios de inclusión se utilizaron los siguientes: padres o tutores con niño menores de 5 años, que conozcan que es un antibiótico, tener una ficha de salud familiar en la unidad de atención primaria y estar de acuerdo en participar. Excluimos a los hogares de la encuesta si los padres o cuidadores no estaban presentes en el momento de la entrevista o si se negaron a participar, si eran profesionales médicos o si no entendieron lo que significaba la palabra "antibióticos".

Para mantener la confidencialidad de la información recolectada no se incluyeron los datos personales tales como nombre y apellido, asegurando la integridad de los participantes. Los datos adquiridos fueron tabulados y analizados estadísticamente mediante el programa "Microsoft Excel versión Office 2016".

\section{Resultados y discusiones}

Gráfica No. 1: Consumo de antibióticos en los últimos 3 meses

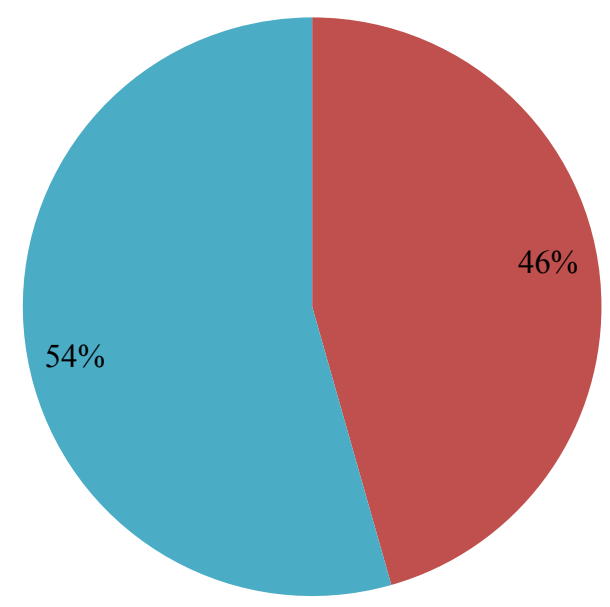

- Pacientes que usaron ATB

- Pacientes que no usaron ATB

Fuente: Contreras C, Almonte C, Martínez P, Valdez C., octubre 2018.

La presente gráfica señala el consumo de antibióticos en los últimos 3 meses en la muestra estudiada. Se observa que el $54 \%$ de la muestra representando 37 encuestados no utilizaron antibióticos durante 
el último trimestre, en contraste con el $46 \%$ que representando 31 encuestados quienes utilizaron antibióticos durante los últimos 3 meses.

\section{Gráfica No. 2: Motivos que requirieron el uso de antibióticos $(\mathrm{N}=68)$}

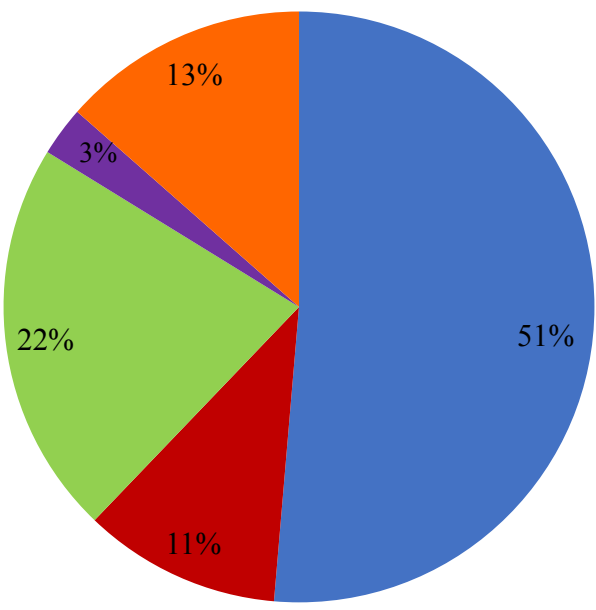

- Aparato Respiratorio

- Aparato Digestivo

$\square$ Piel

- Prematuridad

Procesos Generales e inespecificos

Fuente: Contreras C, Almonte C, Martínez P, Valdez C., octubre 2018.

Esta gráfica muestra las principales circunstancias que requirieron el uso de antibióticos en la muestra estudiada. Mediante la misma se observa que, el $51 \%$ de los niños que consumieron antibióticos se debió a síntomas del aparato respiratorio, $22 \%$ se debió a enfermedades relacionadas a la piel, 13\% correspondió a procesos generales e inespecíficos como fiebre y malestar general, $11 \%$ se debió a situaciones relacionadas al aparato digestivo y solamente un 3\% debido a prematuridad.
Gráfica No. 3: Fuente de adquisición de los antibióticos $(\mathrm{N}=68)$

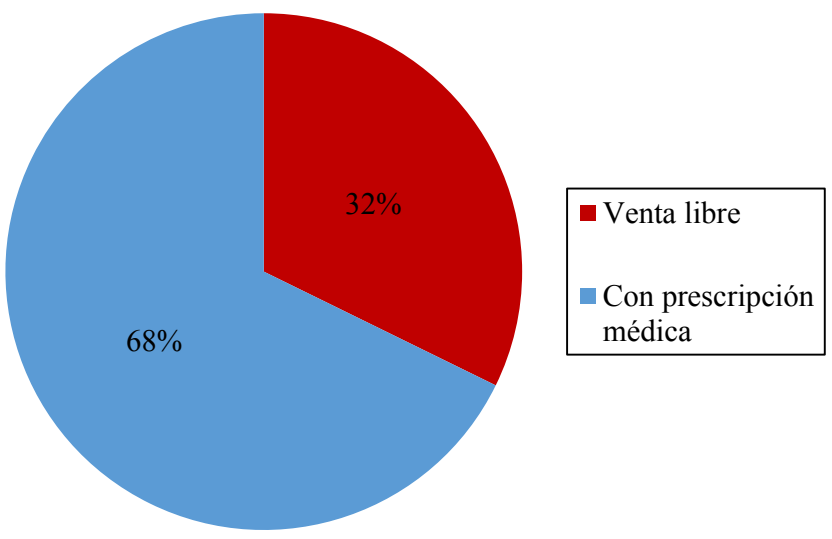

Fuente: Contreras C, Almonte C, Martínez P, Valdez C., octubre 2018.

La presente gráfica muestra el método de adquisición de los antibióticos consumidos. En la misma se tomó en cuenta si los antibióticos fueron adquiridos bajo prescripción médica o por venta libre. Un total de $68 \%$ de los niños consumieron antibióticos bajo prescripción médica contraste con $32 \%$ que consumieron antibióticos por venta libre.

\section{Gráfica No. 4: Antibióticos más utilizados $(\mathrm{N}=68)$}
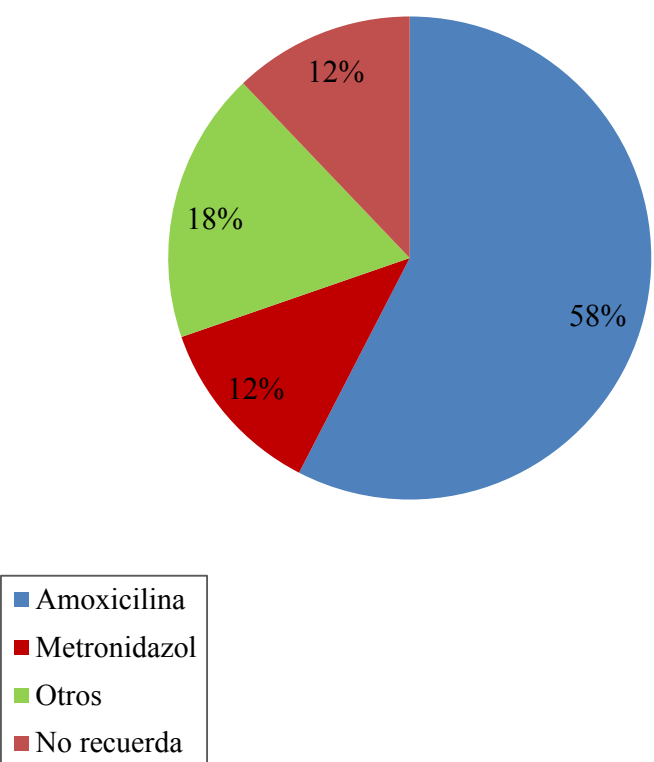

Fuente: Contreras C, Almonte C, Martínez P, Valdez C., octubre 2018. 
Esta gráfica muestra los antibióticos más utilizados en la muestra estudiada. Mediante la misma se observa que, el $58 \%$ de los antibióticos consumidos correspondieron a la Amoxicilina, $18 \%$ correspondió a otros antibióticos dentro de los que estaban Paramomicina, Ceftriaxona, Cefotaxima, Albendazol, Amikacina, Aminosidina y Penicilina Benzatínica. 12\% de los antibióticos consumidos fue representado por el Metronidazol y $12 \%$ de los entrevistados no recordaron los antibióticos que consumieron sus hijos.

\section{Conclusiones}

Del total de la población entrevistada se determinó que a un $54 \%$ de los infantes no se les administró antibióticos durante los últimos 3 meses, en contraste con un $46 \%$ que sí se les administró antibióticos en los últimos 3 meses, estos resultados son distintos a los obtenidos por la investigación "Survey of non-prescribed use of antibiotics for children in an urban community in Mongolia"(7) llevada a cabo por Ganchimeg Togoobaatar y compañeros en el mes de septiembre del año 2010, donde obtuvieron un total de $71 \%$ de infantes a los cuales se le administró antibióticos en los últimos 6 meses, es importante tomar en cuenta que el período delimitado por los mismos es mayor al de esta investigación.

Otras investigaciones han obtenido resultados de uso de antibióticos elevados como una revisión sistemática y metaanálisis publicado en Londres en 2018 donde fueron identificados 3302 artículo, de los cuales 23 estudios cumplieron los criterios de inclusión y se incluyeron en la revisión, concluyó que la proporción general combinada de suministro de antibióticos sin receta fue de un $67 \%$. (8)

De los infantes a los que se le administró antibióticos en los últimos 3 meses, un $68 \%$ correspondió a los indicados por un médico y un $32 \%$ fueron obtenidos por venta libre, en contraste con la investigación de Togoobaatar en la cual un $42.3 \%$ fue adquirido sin prescripción médica en los últimos 6 meses,. Los investigadores atribuyen esta discrepancia al tiempo delimitado de 3 meses versus 6 meses.

Otras investigaciones han resultados en valores similares, según las cuales un menor porcentaje los obtiene sin una prescripción médica como la investigación titulada "Non-prescription use of antibiotics among children in urban China: a cross-sectional survey of knowledge, attitudes, and practices" en la que el $48.2 \%$ de los encuestados informó el uso sin receta de antibióticos para nińos en los últimos 6 meses (9)

Por otro lado, el motivo más frecuente de administración de antibióticos en los infantes, fue por síntomas del aparato respiratorio equivalente a un $51 \%$ y de piel equivalente a un $22 \%$, similar a los resultados obtenidos por Badger-Emeka $\mathrm{L}$ y compañeros en la investigación titulada "Evaluation of the extent and reasons for increased non-prescription antibiotics use in a University town, Nsukka Nigeria" Los resultados mostraron que más del $86 \%$ de los encuestados usan antibióticos no recetados para el tratamiento o la prevención, o ambos. Un porcentaje significativo lo usa para tratar el resfriado y la tos. (10)

El antibiótico más utilizado por los entrevistados fue la amoxicilina con un total de $58 \%$, similar a los resultados obtenidos por los investigadores en Mongolia en el cual la amoxicilina fue el antibiótico más utilizado sin prescripción médica con un total de $58 \%$.

Estos resultados demuestran que en un corto período de tiempo de tan solo 3 meses, menores de 5 años fueron altamente expuestos a antibióticos, debido a que su uso puede contribuir a la resistencia bacteriana, estos resultados tienen implicaciones importantes para la educación en salud pública y para la normativa de ventas de antibióticos en la República Dominicana. 


\section{Recomendaciones}

Estos resultados mostraron implicaciones importantes en la carencia de regulación del uso de antibióticos y el manejo sin conocimientos de la población de esta zona rural, por esto los investigadores recomiendan:

- Que se desarrolle una campańa educativa sobre los riesgos del uso de antibióticos, poniendo especial atención a cómo los usuarios sin receta pueden aportar a incrementar la resistencia bacteriana y los peligros que esta representa.

- Que se organicen talleres a los prestadores de servicio de salud para disminuir las prescripciones innecesarias.

- Que se enriquezca el campo de la investigación ganadera en el tema del uso de antibióticos y sus implicaciones en la salud humana.

- Que se concientice a la comunidad sobre no consumir antibióticos sin prescripción por los riesgos que esta práctica representa.

- Que se preste especial atención a la normativa de ventas de antibióticos en la República Dominicana, y que se transforme, en busca de disminuir el acceso libre de los antibióticos a la comunidad.

\section{Bibliografía}

1. Antimicrobial resistance [Internet]. World Health Organization. 2018 [cited 14 September 2018]. Available from: http:// www.who.int/news-room/fact-sheets/detail/ antimicrobial-resistance

2. Ventola C. The Antibiotic Resistance Crisis: Part 1: Causes and Threats [Internet]. PubMed Central (PMC). 2018 [cited 14 September 2018]. Available from: https://www.ncbi.nlm.
nih.gov/pmc/articles/PMC4378521/

3. Organización mundial de la salud. OMS: Resistencia a los antibióticos. [En línea]. Disponible desde: http://www.who.int/ es/news-room/fact-sheets/detail/resistencia-a-los-antibi\%C3\%B3ticos [Solicitado el 25 de agosto del 2018]

4. Alumran A, Hurst C, Hou X. Antibiotics Overuse in Children with Upper Respiratory Tract Infections in Saudi Arabia: Risk Factors and Potential Interventions. Clinical Medicine and Diagnostics. 2012;1(1):8-16.

5. Organización Mundial de la Salud. OMS: El mundo se queda sin antibióticos. [En línea]. Disponible desde: http://www.who.int/es/ news-room/detail/20-09-2017-the-world-isrunning-out-of-antibiotics-who-report-confirms [Solicitado el 25 de agosto del 2018]

6. Katzunk B, Masters S, Trevor A. Basic and Clinical Pharmacology. Twelfth Edition. New York: McGraw-Hill Medical; 2012

7. Survey of non-prescribed use of antibiotics for children in an urban community in Mongolia. Bull World Health Organ. 2010; 88:930-936

8. Auta A, Hadi M, Oga E, Adewuyi E, AbduAguye $\mathrm{S}$, Adeloye $\mathrm{D}$ et al. Global access to antibiotics without prescription in community pharmacies: A systematic review and meta-analysis. Journal of Infection. 2018.

9. Chang J, Lv B, Zhu S, Yu J, Zhang Y, Ye D et al. Non-prescription use of antibiotics among children in urban China: a cross-sectional survey of knowledge, attitudes, and practices. Expert Review of Anti-infective Therapy. 2018; 16(2):163-172.

10. Badger-Emeka L, Madu Emeka P, Okosi M. Evaluation of the extent and reasons for increased non-prescription antibiotics use in a 
Evaluación del consumo de antibióticos con y sin prescripción médica en infantes menores de 5 años en la comunidad de Paya en medio-Baní, durante el período agosto - octubre 2018

University town, Nsukka Nigeria. International Journal of Health Sciences [Internet]. 2018 [cited 15 September 2018];12(4). Available from: https://www.ncbi.nlm.nih.gov/pmc/articles/PMC6040858/pdf/IJHS-12-11.pdf 